\title{
The root of a comet tail: Rosetta ion observations at comet 67P/Churyumov-Gerasimenko
}

\author{
E. Behar ${ }^{1,2}$, H. Nilsson ${ }^{1,2}$, P. Henri ${ }^{5}$, L. Berčič ${ }^{1,2}$, G. Nicolaou ${ }^{1,4}$, G. Stenberg Wieser ${ }^{1}$, M. Wieser ${ }^{1}$, B. Tabone ${ }^{7}$, \\ M. Saillenfest ${ }^{6}$, and C. Goetz ${ }^{3}$
}

\author{
${ }^{1}$ Swedish Institute of Space Physics, Kiruna, Sweden \\ e-mail: etienne.behar@irf.se \\ ${ }^{2}$ Luleå University of Technology, Department of Computer Science, Electrical and Space Engineering, Kiruna, Sweden \\ 3 Technische Universität Braunschweig Institute for Geophysics and Extraterrestrial Physics, Mendelssohnstraße 3, \\ 38106 Braunschweig, Germany \\ ${ }^{4}$ Mullard Space Science Laboratory, University College London, Dorking, UK \\ 5 LPC2E, CNRS, Orléans, 45071, France \\ 6 IMCCE, Observatoire de Paris, PSL Research University, CNRS, Sorbonne Université, UPMC Univ. Paris 06, LAL, \\ Université de Lille, 75014 Paris, France \\ 7 LERMA, Observatoire de Paris, PSL Research University, CNRS, Sorbonne Université, UPMC Univ. Paris 06, 75014 Paris, France
}

Received 16 February 2018 / Accepted 19 April 2018

\begin{abstract}
Context. The first $1000 \mathrm{~km}$ of the ion tail of comet 67P/Churyumov-Gerasimenko were explored by the European Rosetta spacecraft, 2.7 au away from the Sun.

Aims. We characterised the dynamics of both the solar wind and the cometary ions on the night-side of the comet's atmosphere. Methods. We analysed in situ ion and magnetic field measurements and compared the data to a semi-analytical model.

Results. The cometary ions are observed flowing close to radially away from the nucleus during the entire excursion. The solar wind is deflected by its interaction with the new-born cometary ions. Two concentric regions appear, an inner region dominated by the expanding cometary ions and an outer region dominated by the solar wind particles.

Conclusions. The single night-side excursion operated by Rosetta revealed that the near radial flow of the cometary ions can be explained by the combined action of three different electric field components, resulting from the ion motion, the electron pressure gradients, and the magnetic field draping. The observed solar wind deflection is governed mostly by the motional electric field $-\boldsymbol{u}_{\text {ion }} \times \boldsymbol{B}$.
\end{abstract}

Key words. comets: general - comets: individual: $67 \mathrm{P}$ - plasmas - methods: observational - space vehicles: instruments

\section{Introduction}

All particles originating from a comet's nucleus and subsequently ionised by solar radiation or electron impact eventually escape the comet, reaching on average the velocity of the solar wind. Because of the fast relative speed between the comet and the solar wind, the escaping ions are collimated into a narrow tail, known as the ion tail or gas tail. Contrary to the dust tail, this plasma structure can emit light by fluorescence, and depending on the conditions can be seen from Earth. The shape of these visible ion tails gives a major clue to the existence of both the solar wind and the interplanetary magnetic field (IMF), as for instance elaborated by Alfven (1957). To that extent, the ion tail is one of the major aspects of the interaction between the solar wind and the comet's atmosphere (coma).

During the first part of the 20th century, comets played an important role in the development of space physics. The first suggestion of the existence of a steady stream of charged particles flowing away from the Sun can be attributed to Arthur Eddington (Durham 2006; Eddington 1910), based on observations of comet Morehouse and the analysis of the shapes of the envelopes seen in the atmosphere and in the ion tail. Another model presented by Biermann (1951) describes the tail of comets being dragged by a corpuscular radiation emitted by the Sun. In this description, the momentum is transferred from the wind to the tail by Coulomb collisions. During the same decade, another major model was proposed by Alfven (1957) where the magnetic field of the Sun is 'frozen' in a similar flow of solar particles. The local addition of a cold and slow cometary ion population would correspond to a significant decrease of the total plasma velocity, and the frozen-in magnetic field piles up and drapes itself around the dense coma. This draping pattern was given as an interpretation of the typical pattern of streamers in a comet tail. Comets have acted as natural solar wind probes before the first in situ measurements and the space exploration era because of their high production rate (with values ranging from $10^{25}$ to several $10^{30} \mathrm{~s}^{-1}$ ) and their very low gravity: they were the perfect tracers to make the solar wind 'visible' from Earth.

The first in situ investigation of the interaction between the solar wind and a coma was conducted by the International Cometary Explorer (ICE, launched as ISEE-3, see e.g. Smith etal. 1986) which encountered comet 21P/GiacobiniZinner in September 1985. Since that date, ten other probes have visited eight different comets. ICE, Deep Space 1, and probably Giotto for its second encounter actually flew through the tails of their respective comets, 21P/Giacobini-Zinner, 19P/Borelli, and 
26P/Grigg-Skjellerup, validating some of the theoretical results such as the draping of the IMF remarkably observed by ICE and presented in Slavin et al. (1986). Another noteworthy in situ result is the observation of the tail of comet Hyakutake (C/1996 B2) by the Ulysses spacecraft, 3.8 astronomical units (au) away from the comet's nucleus, reported by Jones et al. (2000). However, these few events are tail crossings; in other words, they only gave a snapshot of the tail structures along one straight line along the spacecraft trajectory. If the coverage along this line is optimum (from outside of the tail to inside to outside again), the spatial coverage along the radial dimension for instance is almost non-existent. The situation for one of the AMPTE mission experiments, the so-called artificial comet, was quite different to these fast passages, and allowed for a more thorough study of how momentum and energy were exchanged between the background incident plasma and the injected heavy ions (Valenzuela et al. 1986; Haerendel et al. 1986; Coates et al. 2015).

At the end of the 22 March 2016, as comet 67P/ChuryumovGerasimenko (67P) was 2.64 au from the Sun and orbiting away from it, the European probe Rosetta operated the first manoeuvre that would bring it on an excursion within the previously unexplored night-side of the coma. The excursion lasted for more than 14 days, during which the spacecraft reached a maximum cometocentric distance of $1000 \mathrm{~km}$, along a complex trajectory (see Fig. 1, upper panel). This was the second and last excursion operated by Rosetta; the first was a day-side exploration conducted in October 2015 during a different activity level and closer to the Sun. An overview of the ion data during the entire mission is shown in Nilsson et al. (2017), where it can also be seen how the ion data from the tail excursion stands out in relation to all the other data.

Contrasting with the otherwise low altitude terminator orbit, this tail excursion allowed the study of the root of the comet's ion tail, and specifically in our case, the study of the plasma dynamics of this region. 67P is less active than most of the previously visited or observed comets (the activity is typically quantified by the production rate of neutral elements). As pointed out by Snodgrass et al. (2017), its ion tail was actually never observed from the ground. To that extent, the interaction between the solar wind and the coma is remarkably different to what is usually described for more active comets closer to the Sun. Instead of the solar wind being deflected symmetrically around the coma and not flowing in the inner region close to the nucleus, we see a coma entirely permeated by the solar particles. By characterising the phase space distribution functions of both the cometary ions and the solar wind, we can understand how the two populations interact in this region, and to what extent this interaction takes part in the escape of cometary ions through the tail, for such a low activity comet far from the Sun. In a statistical study, Berčič et al. (2018) recently described the cometary ion dynamics at the same heliocentric distance, close to the nucleus and in the terminator plane. Two different cometary ion populations were reported, one which gained its energy mostly through its interaction with the solar wind upstream of the nucleus, and one that was accelerated in a region they dominate. That study is put into the perspective of this excursion, completing the larger picture of the interaction. Additionally, Volwerk et al. (2018) present an extensive analysis of the magnetic field observed during the same excursion. The authors found that the magnetic field was not draped around the nucleus in a classical sense; instead, the magnetic field direction was mostly aligned with the IMF direction expected from a Parker spiral model. However, the tail clearly showed a two-lobed structure with regard to the wave activity: directly behind the nucleus the so-called singing comet
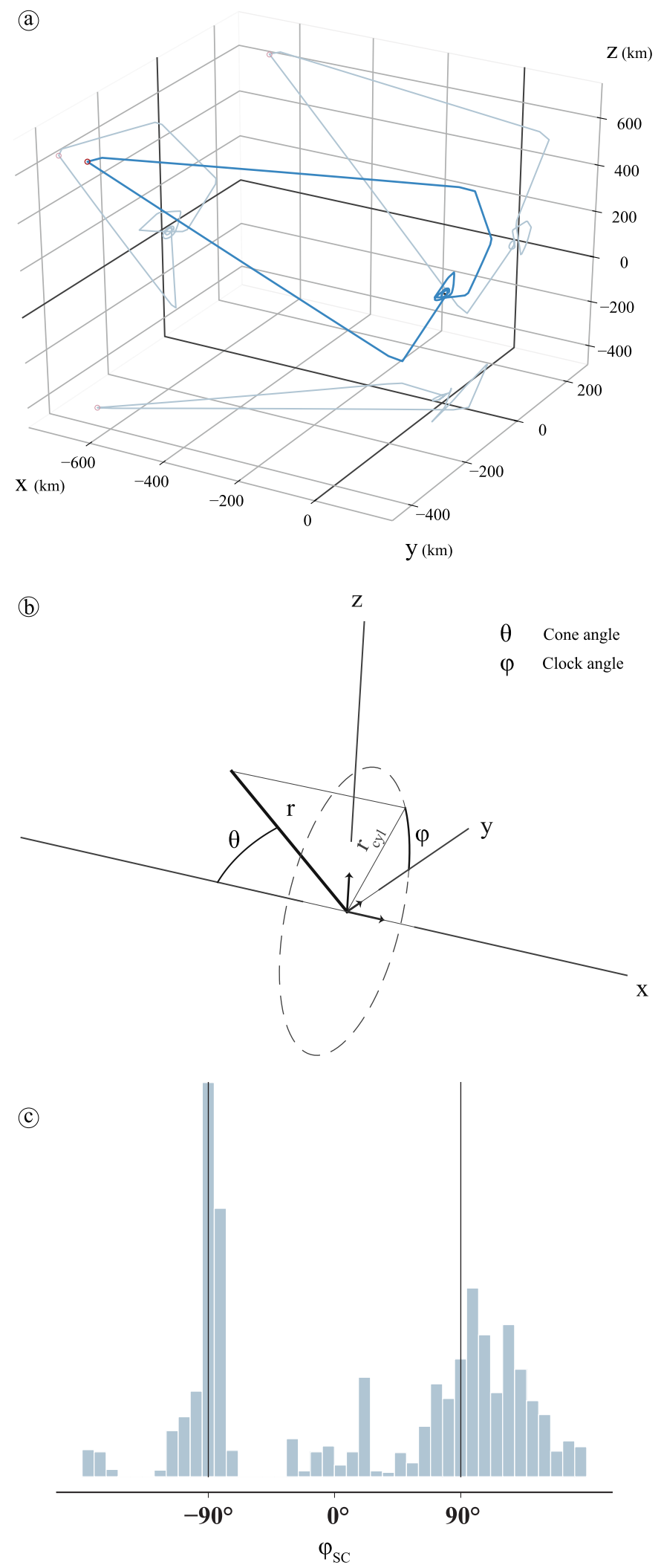

Fig. 1. Panel $a$ : the trajectory of the spacecraft in the CSEQ reference frame (blue) and its projections (grey). Panel $b$ : the cartesian and spherical sets of coordinates. Panel $c$ : the distribution of spacecraft clock angle during the period of interest, in the CSEQ frame.

waves (Richter et al. 2015) were very prominent, whereas to the sides their contribution to the power spectral density becomes negligible. 



Fig. 2. Upper panel: spectrogram of the period, with the considered period displayed by the red arrow. Lower panel: corresponding cometocentric distance $r$ together with the cone angle $\theta$ of the spacecraft.

\section{Instrument and methods}

\subsection{Instrument}

The particle data used in this work were produced by the Ion Composition Analyzer (ICA), part of the Rosetta Plasma Consortium (RPC, Nilsson et al. 2007; Carr et al. 2007). This instrument is an ion mass-energy spectrometer and imager, aimed at studying the interaction between the solar wind and positive cometary ions at comet 67P. The RPC-ICA data consist of count rates given in five dimensions, namely time, energy per charge, mass per charge, and incoming direction (two angles). Full energy scans are produced every $12 \mathrm{~s}$ and full angular scans (corresponding to the full velocity space coverage of the instrument) are produced every $192 \mathrm{~s}$. The energy spans from a few electronvolts up to $40 \mathrm{keV}$ in 96 steps with a resolution $\frac{\delta E}{E}=0.07$. The instrument field of view is $360^{\circ} \times 90^{\circ}$ (azimuth $\times$ elevation), with a resolution of $22.5^{\circ} \times 5.0^{\circ}$. Mass is characterised through an assembly of permanent magnets and a position detection system consisting of 32 anodes, which we will refer to as mass channels. The radial position of ions on the detector plate is a function of both the mass and the energy.

\subsection{Distribution functions \& plasma parameters}

Solar wind ions and cometary ions are well separated in velocity space (i.e. in both energy and direction) and in terms of mass. This is partly illustrated in Fig. 2, which presents a spectrogram (energy versus time) where the different species are identified on the left side of the panel. When the energy separation is poorer, the direction and the mass information allow further identification (see e.g. Nilsson et al. 2015; Berčič et al. 2018). For this
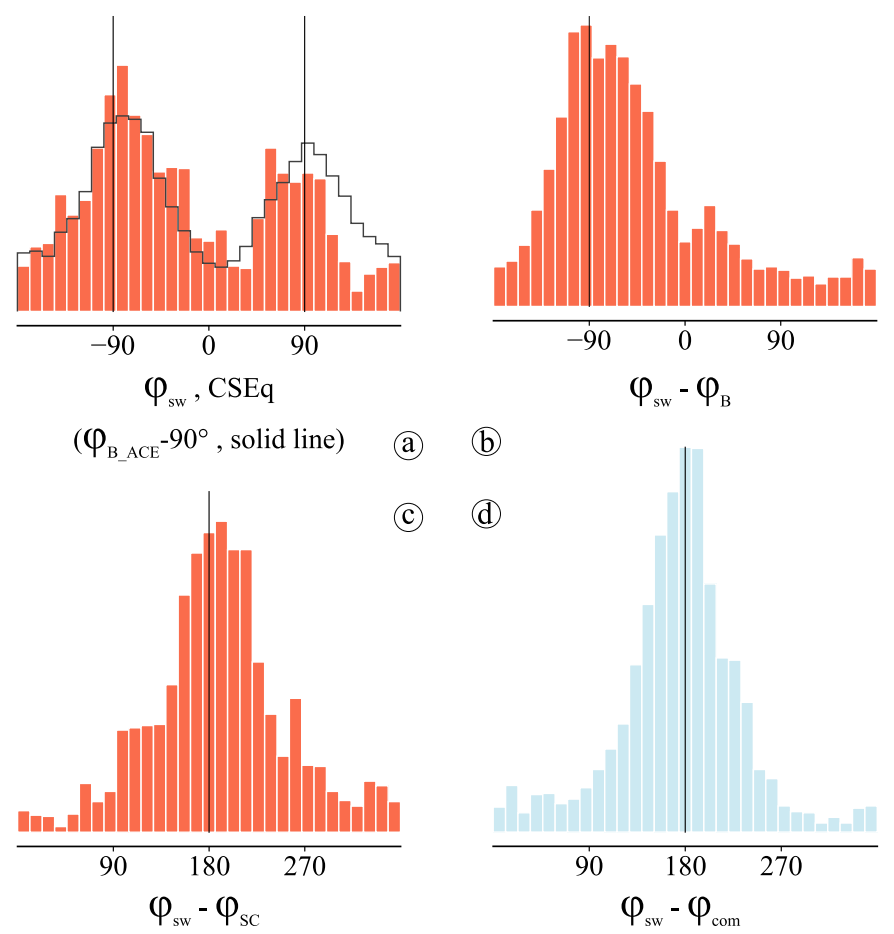

(e) (f)
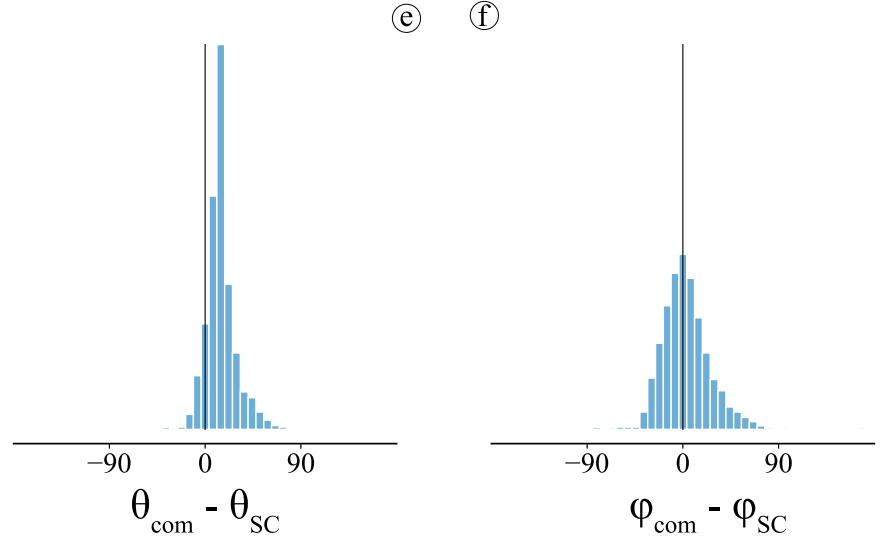

Fig. 3. Panel $a$ : clock angle of the solar wind protons and the estimated upstream magnetic field in the CSEQ frame. Panel $b$ : angular difference (clock angle) between the observed magnetic field and the solar wind protons. Panels $c$ and $d$ : angular difference between the solar wind protons and the spacecraft, and the protons and the cometary ions, respectively. Panels $e$ and $f$ : departure of the cometary ion flow direction from the purely radial direction for the cone and clock angle, respectively.

study, the species were therefore manually selected on daily time intervals.

Using these manual selections, we were able to analyse the velocity distribution functions for each species separately. By integrating the distributions, the plasma moments of order 0 and 1 were calculated, providing the density and the bulk velocity for the different species (Figs. 3 and 5). The distribution functions can be seen in Fig. 4, where red tones are used to represent solar wind protons, and blue tones for cometary ions.

\subsection{Reference frames and coordinate systems}

The bulk velocities were initially calculated in the Cometcentred Solar EQuatorial (CSEQ) frame: the $x$-axis points towards the Sun, and the $z$-axis is oriented by the Sun's north 

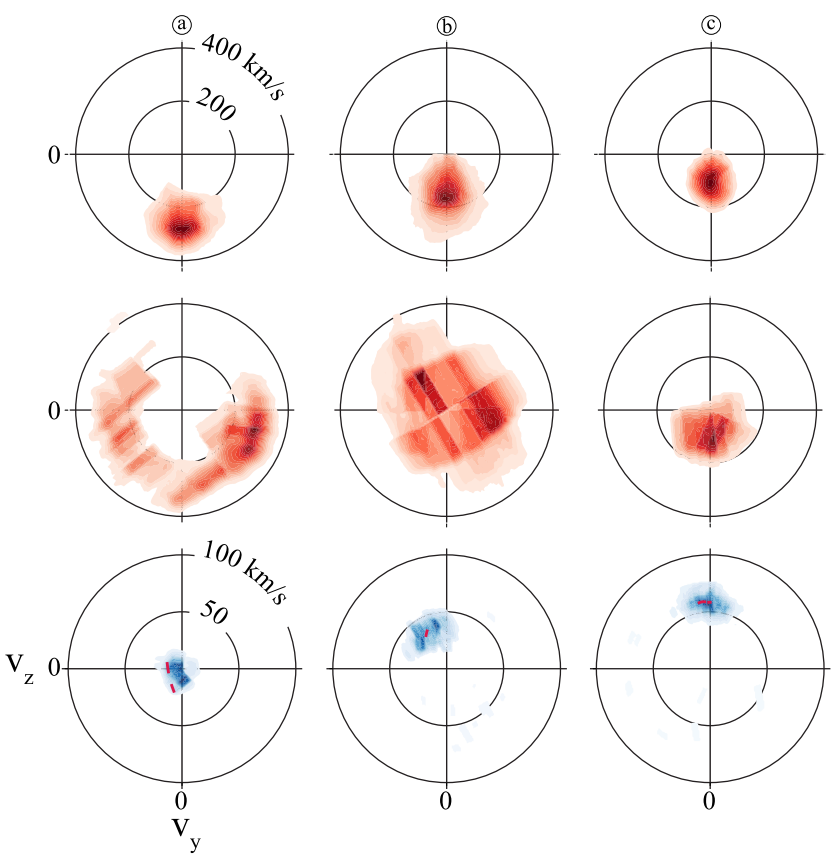

2016-03-27
2016-03-30
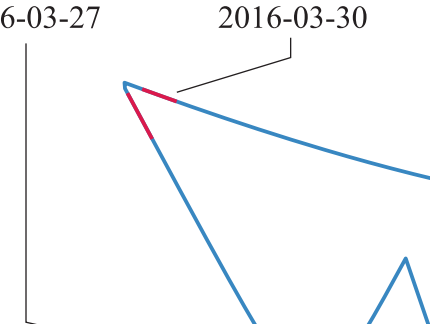

\section{6-04-02}

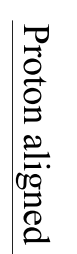

containing the comet-Sun line and the upstream electric field (as observed close to the nucleus in Behar et al. 2016), this rotation leaves the upstream electric field along the $+z$-axis and the upstream magnetic field along the $+y$-axis, in which case this particular frame corresponds to the CSE frame. In the present case, however, this assumption might not be verified, as we discuss below, and this frame can simply be considered a proton-aligned frame.

\subsection{Magnetic fields}

An estimation of the upstream magnetic field is used in the next sections. It corresponds to ACE measurements at the Earth, propagated in time using a ballistic approach accounting for the radial and longitude differences between Earth and 67P, and using the solar wind velocity measured at the Earth. Magnetic field measurements at the spacecraft location are also used, and are provided by the RPC-MAG instrument. A comprehensive description is given in Glassmeier et al. (2007), and a study of the night-side excursion focusing on RPC-MAG results is given by Volwerk et al. (2018).

\section{Results}

\subsection{Overview}

The goal of this study is to characterise and compare the flow of two ion populations in the close tail environment of a comet. This requires the use of numerous combinations of coordinates in order to describe their density, their average direction and average speed, the distribution of these values, and their evolution through time and space as the spacecraft moves along the excursion trajectory. As seen in Fig. 1, part of the excursion is actually on the day-side of the coma, for distances below $500 \mathrm{~km}$. In order to report the entire excursion, this arc is included in the analysis for distances greater than $100 \mathrm{~km}$, adding up to the purely nightside region. The period over which data were analysed in this work - from 23 March to 8 April inclusive - is given by the red arrow below the spectrogram in Fig. 2 .

The first combination of parameters is given in Fig. 2, upper panel, and is the combination of time, energy, and number of detections of all ions. Four different species are identified on the left-hand side of the panel, a group of three solar wind species (protons $\mathrm{H}^{+}$, alpha particles $\mathrm{He}^{2+}$, and singly charged helium particles $\mathrm{He}^{+}$, which result from charge exchange between the cometary neutral atmosphere and solar wind alpha particles, see Nilsson et al. 2015) and the cometary ions, assumed to be dominated by water molecules $\mathrm{H}_{2} \mathrm{O}^{+}$. The evolution of the spacecraft cometocentric distance is given in the lower panel, together with the cone angle of the spacecraft $\theta_{\mathrm{SC}}$. One of the most striking results is already seen here, namely the correlation between the average energy of the cometary ions and the cometocentric distance. Their detection rate also seems to decrease with the cometocentric distance (as confirmed later on). In opposition, no obvious effect on the solar wind can be found in this figure (with variations in the average energy, the energy width, and the particle counts being in the usual range of fluctuations either of upstream origin or from the general interaction between the solar wind and the coma).

\subsection{Flow directions}

The bulk velocity directions of solar wind and cometary ions are summarised in Fig. 3. The first three histograms a, b, and c (2018). With the assumption that protons are deflected in a plane 
Behar, E. et al.: The root of a comet tail: Rosetta ion observations at comet $67 \mathrm{P} /$ Churyumov-Gerasimenko
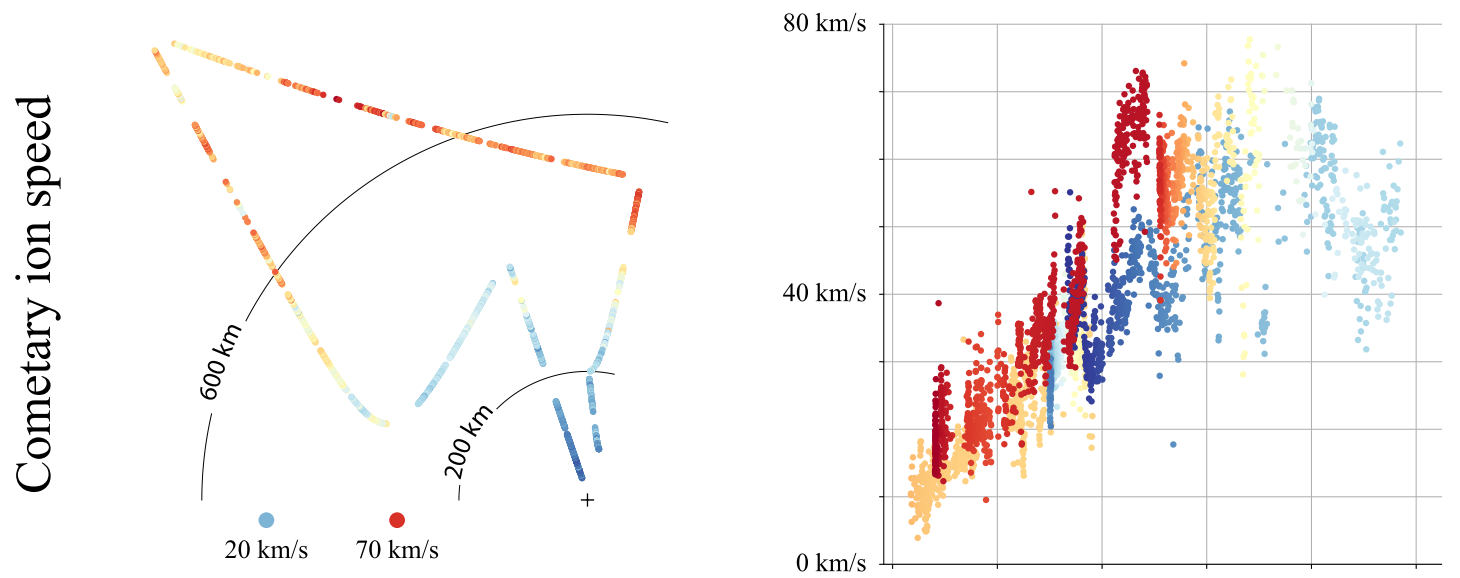

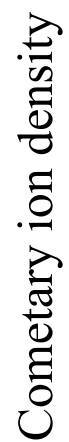
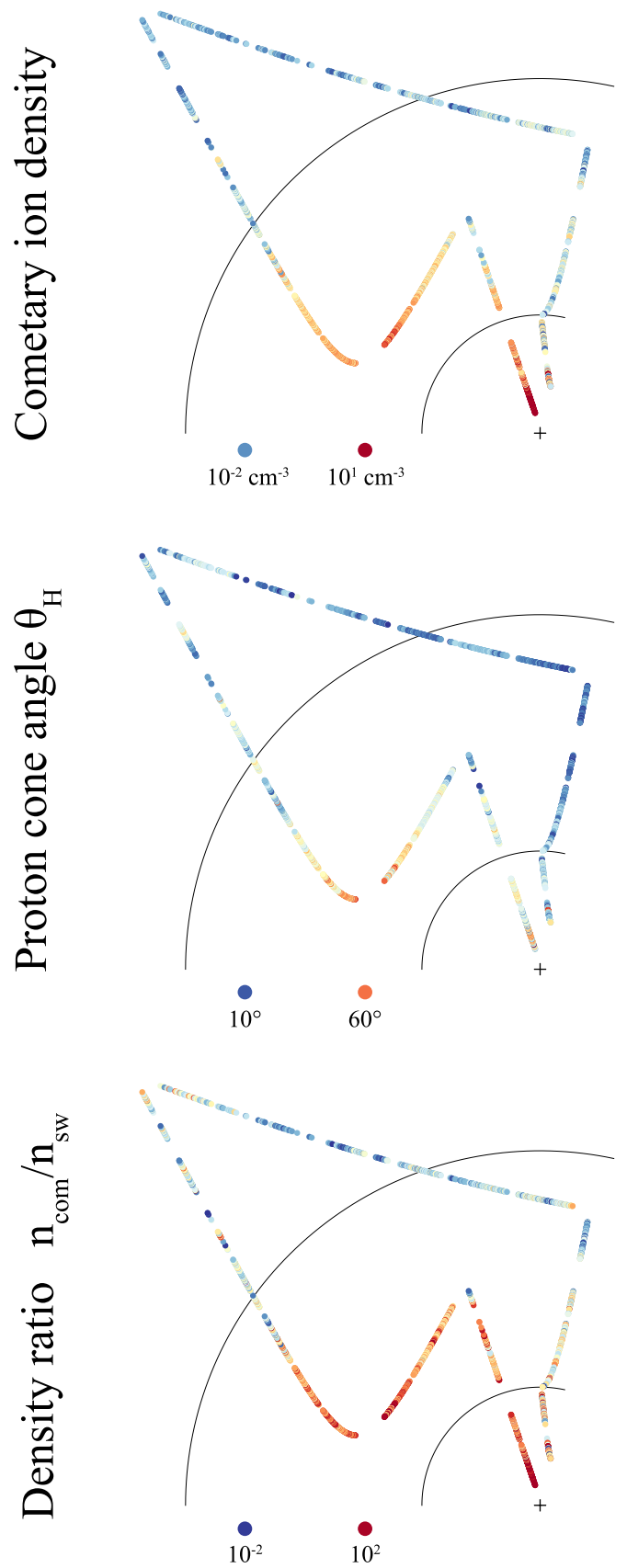
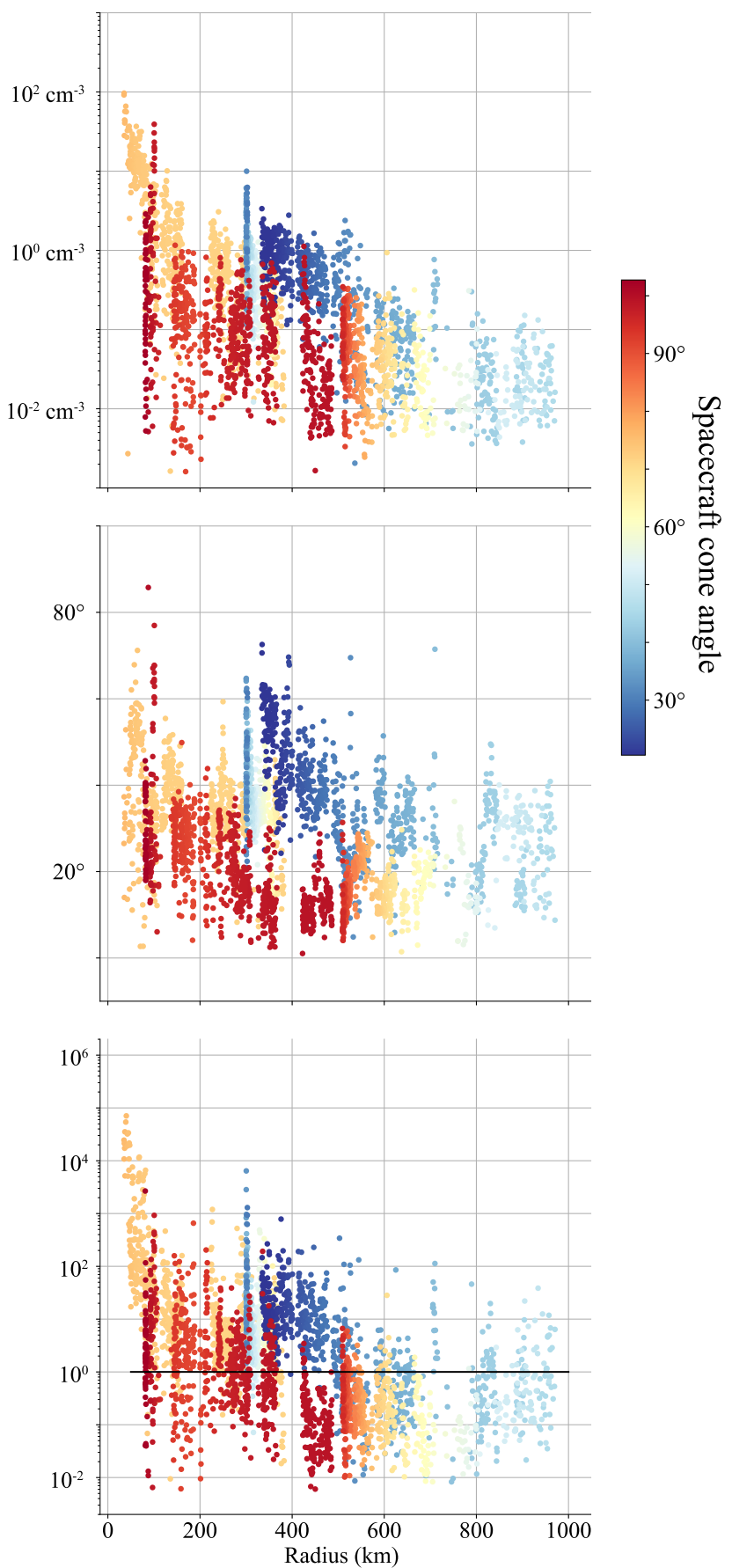

Fig. 5. Plasma parameters for cometary and solar wind ions. 
describe the proton flow direction in terms of clock angle ${ }^{1}$. In (a) they are seen deflected away from the $-x$-direction with a corresponding clock angle peaking on average at either $-90^{\circ}$ or $+90^{\circ}$ in the CSEQ reference frame. The same clock angle is on average $-90^{\circ}$ away from the magnetic field clock angle measured at the spacecraft, histogram $b$, even though all configurations were observed (breadth of the distributions). The spacecraft trajectory also presents two peaks in its clock angle distribution, at $-90^{\circ}$ and $+90^{\circ}$, in Fig. 1 bottom histogram, similarly to protons. In turn, the difference of the two clock angles (Fig. 3c) shows a very clear trend centred on $180^{\circ}$, with $56 \%$ of the occurrences between $135^{\circ}$ and $225^{\circ}$.

Next, we examine the departure of the cometary ion flow from the purely radial direction, i.e. the direction opposite to that of the nucleus seen from the spacecraft. The difference of cone and clock angles are given by the two histograms e and $\mathrm{f}$, and display much narrower distributions than previously. The distribution of the cone angle difference peaks at a bit more than $10^{\circ}$ (the particles are leaning from the radial to the antisunward direction), and the distribution of the clock angle difference has its maximum value at $0^{\circ}$ on average, cometary ions were seen flowing more or less radially away from the nucleus.

The solar wind proton and cometary ion mean flow directions are compared in histogram $\mathrm{d}$. They are seen on average flowing $180^{\circ}$ apart from each other in terms of clock angle, with once again a very broad distribution presenting all configurations.

\subsection{Distribution functions}

To go further than the bulk direction of the flows and diagnose the actual spread of the populations in velocity space, the velocity distribution functions obtained during the three different days are shown in Fig. 4. The projection of the distributions in the plane $\left(v_{y}, v_{z}\right)$ is displayed for cometary ions (blue) and solar wind protons (red) in the CSEQ frame (middle and bottom rows), and in the proton-aligned frame (top row). Data were integrated over a period of about $15 \mathrm{~h}$ in each case, displayed on the orbit as the red segments (the orbit is projected in the $(r, \theta)$-plane). The three dates are chosen to illustrate the extreme cases: the first is taken from the inner region closest to the comet-Sun line, the second is taken from the farthest explored cometocentric distance, and the last corresponds to the region slightly day-side, close to the terminator plane, $500 \mathrm{~km}$ from the nucleus. These segments are cut in two parts because of data gaps matching spacecraft manoeuvres.

In the cometary ion distributions (bottom panels, blue), an indication of the radial velocity is given by the red lines: it is the velocity of particles that would flow purely radially away from the nucleus with the same average speed as the observed cometary ions. Because of the slow motion of the spacecraft, these lines are very limited.

The cometary population is well focused, beam like, in the CSEQ frame, even integrated over 15 hours. The distributions are also well focused along $v_{x}$ (not shown here). In the same frame, the solar wind protons have much broader velocity distributions for the two first cases a and b. However, the last set of distributions (Fig. 4c) presents the average case seen in the histogram d of Fig. 3: two beams (cometary and solar wind) flowing $180^{\circ}$ apart in the $\left(v_{y}, v_{z}\right)$-plane, with cometary ions purely radial.

\footnotetext{
1 Only clock angles corresponding to a cone angle greater than $10^{\circ}$ are considered to avoid poorly determined clock angles.
}

When aligning the bulk velocity of the solar wind protons with the $v_{y}$-axis for each $192 \mathrm{~s}$ scan, we get a well-focused beam for each day, which proves that the solar wind remains a beam at any time, only deflected and changing direction in the CSEQ frame, associated with rotations of the upstream magnetic field clock angle (Behar et al. 2016). It was verified that the rotation also focuses the proton distributions along the $v_{x}$ dimension (not shown here).

\subsection{Cometary ion speed}

The cometary ion speed is given in Fig. 5a as the norm of the bulk velocity. The right-hand panel gives the evolution of the speed with the cometocentric distance $r$, and the spatial distribution measured along the spacecraft trajectory is given in the left-hand panel. The first obvious observation is that cometary ions gain speed until a distance to the nucleus of about $600 \mathrm{~km}$. Further out, the observed speed is much more spread out, and no clear trend is found.

\subsection{Cometary ion density}

The density of particles originating from the nucleus is given in Fig. 5b, showing a reduction of around three orders of magnitude between closest and furthest point from the nucleus. Further away than $600 \mathrm{~km}$ from the nucleus, the density seems to flatten out with values between 1 and 0.1 particle $\mathrm{cm}^{-3}$.

\subsection{Solar wind proton deflection}

Features of cometary origin in the solar wind speed and density could not be separated from the typical upstream variations in the observations during the excursion. However the solar wind deflection, i.e. the cone angle of the solar wind proton velocity $\theta_{\mathrm{sw}}$, shows a clear spatial evolution. In Fig. $5 \mathrm{c}$, we can see that for high cone angles and high radii, this deflection can be as low as $10^{\circ}$, whereas for spacecraft cone angles below $45^{\circ}$ and radii below $600 \mathrm{~km}$ the deflection reaches $50-60^{\circ}$. The distribution functions in Fig. 4 illustrate low and high deflection cases, and show how beam-like the distributions remain (in the proton-aligned frame). Such a value of $50^{\circ}$ is well in line with the average deflection angles of that period, measured on the terminator plane close to the nucleus, before and after excursion. These average values can be seen in the data presented in Behar et al. (2017, Fig. 1) for both the alpha particles and the protons. In particular, the decrease corresponding to the night-side excursion can be easily recognised.

\subsection{Solar wind ions versus cometary ions}

The charge density ratio between the cometary ions and the solar wind is given in Fig. $5 \mathrm{~d}$ as $n_{\mathrm{com}} / n_{\mathrm{sw}}$. The colour map displays a dominant cometary population as red tones and a dominant solar wind population as blue tones, whereas ratios close to 1 are displayed as pale yellow tones. Cometary ions dominate in terms of charge density $63 \%$ of the measurements.

The density ratio plotted against the radius (right-hand panel) shows a visible correlation with the cometary ion density and the solar wind deflection. The solar wind density variations are thus much smaller than those of the cometary ion density, and do not play an important role in the evolution of this ratio. The correlation with the solar wind deflection is discussed in the next section. 


\section{Discussion}

\subsection{Cometary ion origin}

During the excursion, cometary ions are observed flowing mostly radially at all locations. It is fairly safe to believe that they originate from a region close to the nucleus. In Berčič et al. (2018), the authors made a statistical analysis of the cometary ion dynamics for typical orbit conditions, i.e. low cometocentric distance (about $30 \mathrm{~km}$ ) and within the terminator plane. The heliocentric distance turns out to be the same for this statistical study (between $2.5 \mathrm{au}$ and $2.7 \mathrm{au}$ ) and for the present excursion, which allows a direct comparison between the average cometary ion behaviour at the terminator plane, close to the nucleus, and their behaviour in the night-side region. The major result of Berčič et al. (2018) is the characterisation of two main cometary populations, namely the pick-up and the expanding populations. Whereas the pick-up ion population (average speed $30 \mathrm{~km} \mathrm{~s}^{-1}$ ) is well ordered by the upstream solar wind electric field and originates from the day-side of the coma, the expanding population (on average about $6 \mathrm{~km} \mathrm{~s}^{-1}$ ) presents a cylindrical symmetry around the comet-Sun line in terms of flow direction. However, as observed $30 \mathrm{~km}$ away from the nucleus, these expanding ions are not moving purely radially, and have an additional anti-sunward component. As presented by the same authors, the acceleration of this population could in part result from a radial ambipolar electric field, set up by charge separation between fast moving electrons and slower ions, due to pressure gradients of the spherically outflowing atmosphere. The cometary ions observed further out along the excursion, much faster than the expanding population, were once part of it. The extent of the region in which the ambipolar electric field may dominate has not been investigated yet, and does not appear as a sharp boundary in the present results. Far from trivial, this topic would most likely require the use of self-consistent fully kinetic numerical models.

\subsection{Spacecraft position}

On average and in the equatorial plane of the Sun, the IMF is twisted in a Parker spiral, pointing either outward or inward. This average configuration corresponds to an IMF clock angle of respectively $0^{\circ}$ or $180^{\circ}$ in the CSEQ reference frame, which is perfectly seen in the propagated ACE data, Fig. 3a. The corresponding upstream solar wind electric field is therefore on average along the $z$-axis, i.e. a clock angle of respectively $-90^{\circ}$ and $+90^{\circ}$. The deflection of the solar wind was observed during the entire mission and has been shown to be the result of the mass-loading occurring upstream of the measurement point, in a region dominated by the solar wind: the new-born cometary ions, with almost no initial velocity, are accelerated by the local motional electric field. The momentum and energy they gain is taken from the solar wind, which in turn is necessarily deflected with a clock angle opposite to that of the cometary ions (see e.g. Behar et al. 2016; Berčič et al. 2018), therefore $-90^{\circ}$ away from the magnetic field clock angle. In Fig. 3, histogram a, the propagated ACE data are shifted by $-90^{\circ}$, and overplotted (solid black line) with the proton clock angle in the same frame. A very nice match is found, in shape and even in the relative hight of the two peaks. Measured at the spacecraft, the magnetic field is on average $-90^{\circ}$ from the proton clock angle as well, as seen in histogram b. From histograms a, b, and d of Fig. 3, it appears that on average, the proton flow direction observed during the excursion is compatible with the effect of the mass-loading happening upstream of (and all the way to) the measurement point.

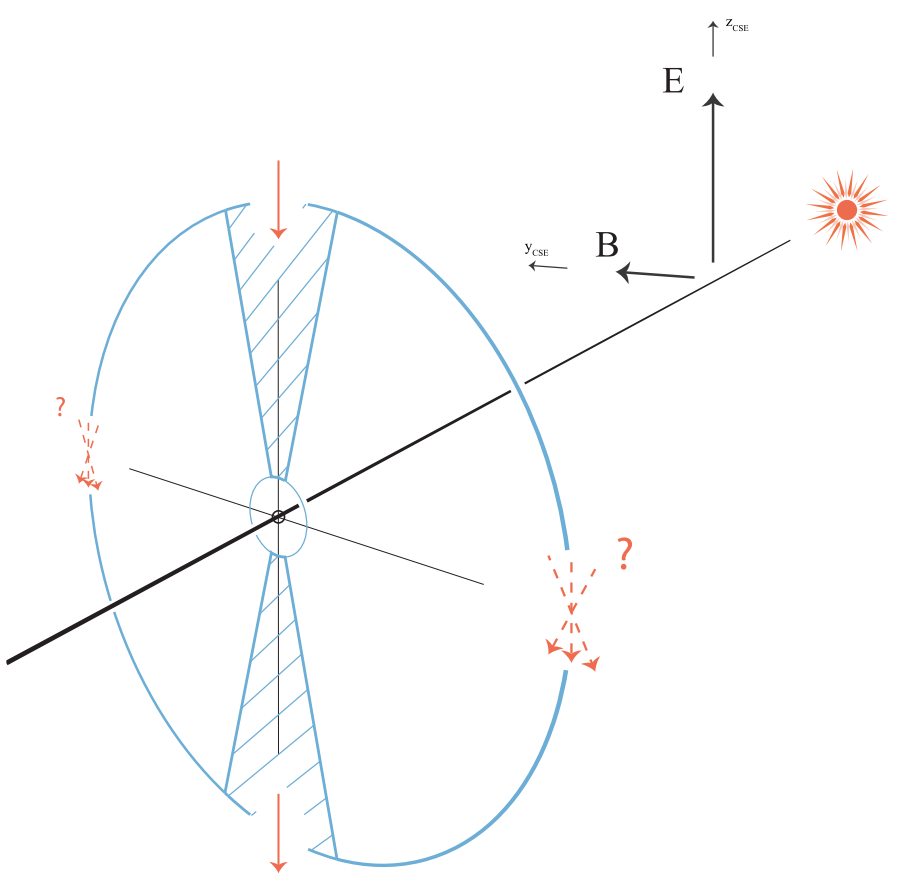

Fig. 6. Representation of the CSE frame, and the regions where the selected data are estimated to be measured (striped). The red arrows indicate the expected velocity of solar wind protons, projected in the plane $\left(y_{\mathrm{CSE}}, z_{\mathrm{CSE}}\right)$. The nucleus position is not shown as it is not fixed on this representation.

Assuming to first order that the solar wind protons are deflected with a clock angle of $-90^{\circ}$ everywhere in the CSE frame of reference, then a clock angle difference of $180^{\circ}$ between the protons and the spacecraft position in CSEQ implies that the spacecraft had no $y$-component and a positive $z$-component in CSE. For a difference of $0^{\circ}$, the spacecraft has no $y$-component and a negative $z$-component. This is illustrated in Fig. 6 by the two striped regions. This is the case most of the time during the excursion. However, the proton velocity can also gain a $y$-component, due to the total electron pressure gradients, magnetic field draping and upstream magnetic field cone angle (angle of the average Parker spiral). In this case, represented by dashed arrows in Fig. 6, the CSE and the proton aligned frames are no longer equivalent. However, in the plane $y_{\mathrm{CSE}}=0$ this $y$-component is close to zero because of the general symmetry of the draping pattern and the pressure (see respectively Alfven 1957 and Haser 1957). Additionally, the angle of the Parker spiral at this distance to the Sun is around $70^{\circ}$ from the comet-Sun line, which is fairly close to $90^{\circ}$, a value for which the ideally draped field lines are perfectly symmetric. Therefore, in summary, a clock angle difference close to $180^{\circ}$ or $0^{\circ}$ is still a valid indication of the spacecraft position in the CSE frame. This is illustrated in the schematic in Fig. 7. As seen in the previous section, almost $60 \%$ of the observations are estimated to be within $\pm 45^{\circ}$ of the $(y=0)$ plane.

\subsection{The interaction}

This subsection is extensively based on the global 2D model of the interaction between the solar wind and the coma given in Behar et al. (2018), and the analysis of its dynamics given by Saillenfest et al. (2018). We summarise the main aspects of the model before comparing it to the observations. 
In a cometary environment, all forces but the Lorentz force $\boldsymbol{F}=e(\boldsymbol{E}+\boldsymbol{v} \times \boldsymbol{B})$ (with the electric field $\boldsymbol{E}$, the magnetic field $\boldsymbol{B}$, and the velocity $\boldsymbol{v}$ and the single charge $e$ of the considered particle), can be neglected. The alpha particles are also neglected, as well as pressure gradients, collisions, and electron inertia. Furthermore, considering spatial scales $\ell$ much greater than the ion inertial length $d_{\mathrm{i}}$, the total electric field is simplified to its ideal MHD form, hereafter referred to as $\boldsymbol{E}_{\text {motional }}$. Considering two plasma beams (one solar wind proton beam and one cometary ion beam), we have

$\boldsymbol{E}_{\text {motional }}=-\boldsymbol{u}_{\text {ion }} \times \boldsymbol{B}$

$=-\left(\frac{n_{\mathrm{sw}}}{n_{\mathrm{sw}}+n_{\mathrm{com}}} \boldsymbol{u}_{\mathrm{sw}}+\frac{n_{\mathrm{com}}}{n_{\mathrm{sw}}+n_{\mathrm{com}}} \boldsymbol{u}_{\mathrm{com}}\right) \times \boldsymbol{B}$.

The corresponding Lorentz force on each species then reads

$\boldsymbol{F}_{\mathrm{sw}}=e \frac{n_{\mathrm{com}}}{n_{\mathrm{sw}}+n_{\mathrm{com}}}\left(\boldsymbol{u}_{\mathrm{sw}}-\boldsymbol{u}_{\mathrm{com}}\right) \times \boldsymbol{B}$
$\boldsymbol{F}_{\mathrm{com}}=-e \frac{n_{\mathrm{sw}}}{n_{\mathrm{sw}}+n_{\mathrm{com}}}\left(\boldsymbol{u}_{\mathrm{sw}}-\boldsymbol{u}_{\mathrm{com}}\right) \times \boldsymbol{B}$.

The model then focuses on the plane $\left(y_{\mathrm{CSE}}=0\right)$. The symmetry of this ideal system (as mentioned in the previous section) gives in this plane $\boldsymbol{B}=B_{y} \hat{\boldsymbol{y}}$. Assuming that $u_{\mathrm{sw}} \gg u_{\text {com }}$, we get

$\boldsymbol{F}_{\mathrm{sw}}=e B \frac{n_{\mathrm{com}}}{n_{\mathrm{sw}}+n_{\mathrm{com}}} \boldsymbol{u}_{\mathrm{sw}} \times \hat{\boldsymbol{y}}$
$\boldsymbol{F}_{\mathrm{com}}=-e B \frac{n_{\mathrm{sw}}}{n_{\mathrm{sw}}+n_{\mathrm{com}}} \boldsymbol{u}_{\mathrm{sw}} \times \hat{\boldsymbol{y}}$.

By considering only new-born cometary ions flowing radially away from the nucleus at the speed $u_{0}$, and neglecting the accelerated (or pick-up) cometary ions, the dynamics of the protons is reduced to

$\boldsymbol{F}_{\mathrm{sw}}=\frac{m_{\mathrm{sw}} \eta}{r^{2}} \boldsymbol{u}_{\mathrm{sw}} \times \hat{\boldsymbol{y}} ; \quad \eta=\frac{e v_{i} Q B_{\infty}}{4 \pi v_{\mathrm{ml}} n_{\mathrm{sw}} m_{\mathrm{sw}} u_{0}} \quad\left[\mathrm{~m}^{2} / \mathrm{s}\right]$,

where $Q$ is the production rate of neutral elements, $v_{i}$ is the ionisation rate (taken to be constant through the coma), $B_{\infty}$ is the amplitude of the upstream magnetic field, and $n_{\mathrm{sw}}$ the density of the solar wind, also assumed to be constant at zero order. The parameter $v_{\mathrm{ml}}$ is a non-physical destruction rate of cometary ions, which allows us to neglect the accelerated cometary ions in the analytical expression of the cometary ion density. All the previous values are taken from the literature, and $v_{m l}$ is estimated to be about $0.01 \mathrm{~s}^{-1}$ based on the values found in Behar et al. (2016). When compared with the data of the excursion, $v_{\mathrm{ml}}$ is taken as a free parameter to allow a better fit to the data and to allow it to absorb the uncertainty of all other parameters. However, its final value is found to be in this precise range.

As seen in Eq. (4), the solar wind protons experience a force always orthogonal to their velocity and with an amplitude proportional to $1 / r^{2}$. Protons are not decelerated and are only deflected. These dynamics have been thoroughly analysed by Saillenfest et al. (2018). The resulting proton trajectories are given in Fig. 7, bottom-left panel, as red lines. One characteristic of the dynamics is the formation of a caustic, along which proton trajectories intersect, a structure also observed in numerical models (see Behar et al. 2018 for a discussion of numerical models). In the resulting flow lines, two types of trajectories are to be considered with caution. After passing the caustic, the trajectories of protons are unphysical as they are not aligned with the local bulk velocity: they are expected to gyrate in a more complex manner. Additionally, the region downstream of the caustic for $z_{\mathrm{CSE}}<0$ is poorly modelled, mostly because of the outflow from the caustic (absent in this model), and the pile-up of the magnetic field is expected to be significantly more complex than modelled in this precise region (see Behar et al. 2018 for more details). Accordingly, these two types of trajectories are lightened in Fig. 7.

As previously discussed, many observations are estimated to be taken close to the $\left(y_{\mathrm{CSE}}=0\right)$ plane. From histogram $\mathrm{c}$ in Fig. 3, we have selected the data points lying in the interval $\left[160^{\circ}, 200^{\circ}\right]$ (the peak of the distribution) and in the intervals $\left[0^{\circ}, 20^{\circ}\right]$ and $\left[340^{\circ}, 360^{\circ}\right]$, the tips of the wings of the distribution. These data represent $33 \%$ of all the valid data. They are estimated to have been taken within $\pm 20^{\circ}$ of the plane $\left(y_{\mathrm{CSE}}=0\right)$, which we illustrate with the two striped surfaces in the schematics of Fig. 6. The velocity vectors of the selected data are projected in the cartesian $(x, z)$-plane of the estimated CSE frame of reference. The proton velocity vectors are compared with the 2D model, and comparison for a value of $v_{\mathrm{ml}}=0.01 \mathrm{~s}^{-1}$ is given in Fig. 7, lower left panel, in the lower range of the interval estimated in Behar et al. (2018).

For $z_{\mathrm{CSE}}>0$ and $r>200 \mathrm{~km}$, a very good agreement between the observed proton bulk velocity and the modelled proton flow direction is found. The data at $z_{\mathrm{CSE}}<0$ lie in the region poorly constrained by the model, with all data points downstream of the caustic. We note that a higher value of the rate $v_{\mathrm{ml}}$ corresponding to an overall smaller deflection of the solar wind would give a better fit for data points for $r<200 \mathrm{~km}$. It appears that the model together with a value of $v_{\mathrm{ml}}$ of about $0.01 \mathrm{~s}^{-1}$ accounts for the general behaviour of the solar wind close to the plane $\left(y_{\mathrm{CSE}}=0\right)$ as seen during the night-side excursion.

The model does not solve the dynamics of the cometary ions. However, it gives us a valuable hint through the expression of the force they experience:

$\boldsymbol{F}_{\mathrm{com}}=-e \frac{n_{\mathrm{sw}}}{n_{\mathrm{sw}}+n_{\mathrm{com}}}\left(\boldsymbol{u}_{\mathrm{sw}}-\boldsymbol{u}_{\mathrm{com}}\right) \times \boldsymbol{B}$

For $54 \%$ of the selected observations, cometary ions are at least one order of magnitude slower than the solar wind protons. Therefore, $\boldsymbol{F}_{\text {com }}$ is fairly orthogonal to the proton velocity. But we can go further, and can calculate either the motional electric field or the force experienced by the cometary ions since all quantities are actually measured by RPC-ICA and RPC-MAG. The result for $\boldsymbol{E}_{\text {motional }}$ is given in Fig. 7 in the upper right panel. It represents the indirect measurement of that field, independent of any assumptions. The norm of the vectors depends on the density and velocity of particles and on the magnetic field amplitude, but the direction of the vectors only depends on the velocity vectors direction and the magnetic field direction. The RPC-ICA sensor is better at measuring the velocity of particles than their density; therefore, the direction of the vectors is better constrained than their norm. It should also be noted, however, that magnetic field data are not ideally constrained for most of the time interval, as explained in Volwerk et al. (2018). Based on Fig. 3b, we assumed the magnetic field to be orthogonal to the protons, which makes the computed electric field amplitude an upper limit.

The motional electric field results in a higher cometary ion acceleration further away from the nucleus in a region where the solar wind tends to dominate (Fig. 5, bottom panel). The corresponding force is aligned with the flow direction in the $z>0$ region for small $x$-coordinates. Going down in the inner tail region, the cometary ions are seen gradually departing from the field direction, to end up being completely misaligned for $z<0$. Obviously, other sources of electric field are at work there. It is noteworthy that in this region, the singing comet waves in the 
Behar, E. et al.: The root of a comet tail: Rosetta ion observations at comet 67P/Churyumov-Gerasimenko

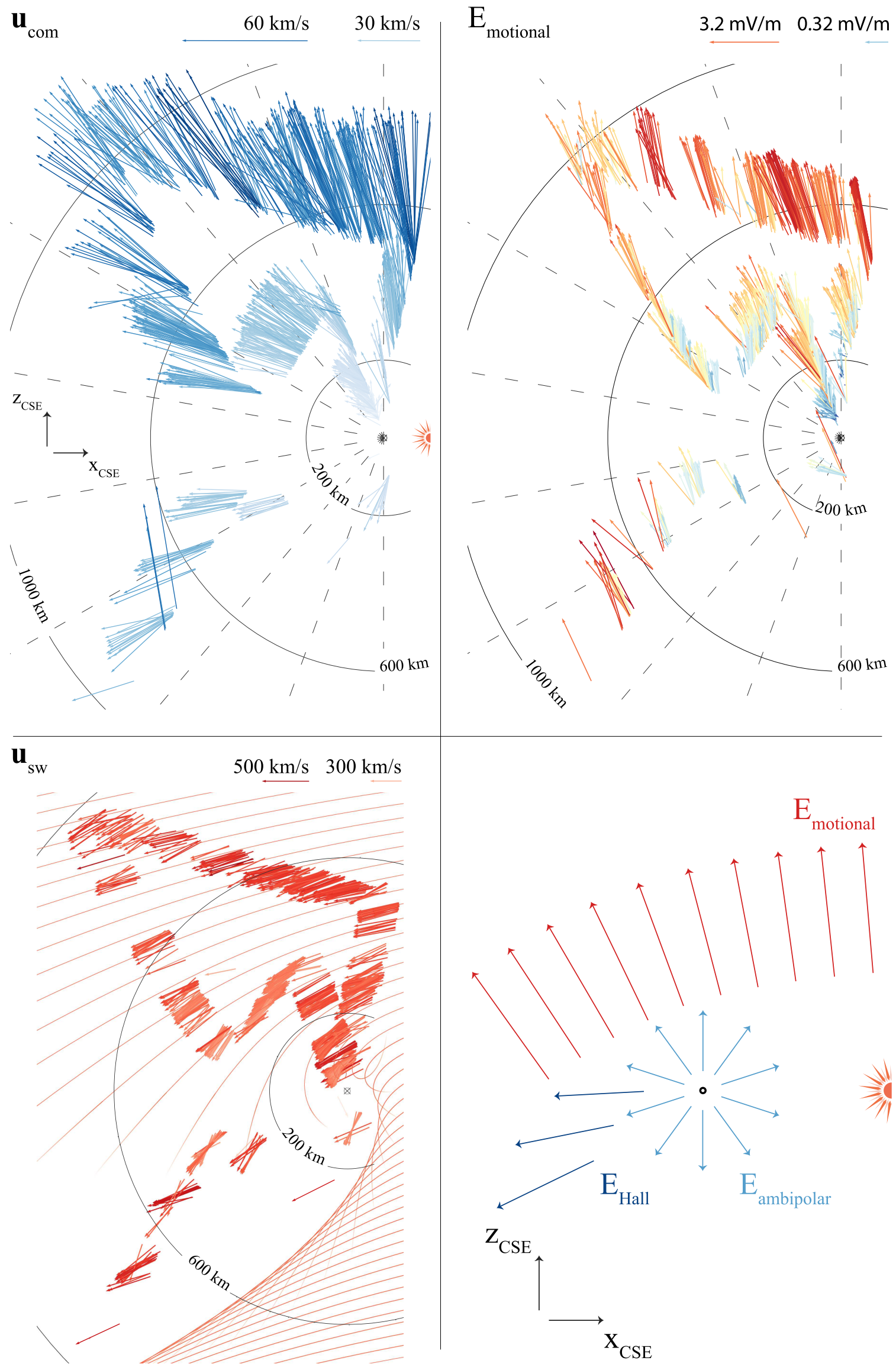

Fig. 7. Upper left: observed cometary ion bulk velocities in CSE. Lower left: observed solar wind proton bulk velocities in CSE, with their theoretical trajectories. Upper right: indirectly observed motional electric field. Lower right: schematics of the three terms of the total electric field (interpretation). 
magnetic field (Richter et al. 2015) were found to be stronger than further out on the night-side of the coma, as shown by Volwerk et al. (2018).

\subsection{Hall electric field \& pressure gradients}

The generalised Ohm's law, when neglecting collisions and neglecting the electron mass compared to the ion mass, is reduced to

$\boldsymbol{E}=-\boldsymbol{u}_{\text {ion }} \times \boldsymbol{B}+\frac{1}{n e} \boldsymbol{j} \times \boldsymbol{B}-\frac{1}{n e} \nabla P_{e}$

with $\mathbf{j}$ the total current, $\nabla P_{\mathrm{e}}$ the electron pressure gradient, and $n=n_{\text {sw }}+n_{\text {com }}$ the total ion density. We have previously investigated the first of the three terms on the right-hand side of the equation, namely the motional electric field $\boldsymbol{E}_{\text {motional }}$. Neglecting the displacement current $\partial_{t} \boldsymbol{E}$ in the AmpèreMaxwell law, the second one - the Hall term - becomes $\boldsymbol{E}_{\text {Hall }}=(\nabla \times \boldsymbol{B}) \times \boldsymbol{B} /\left(\mu_{0} n e\right)$. It can arise from the magnetic field draping around the coma, which is a source of curl for $\boldsymbol{B}$. Using Ampère's law, we can find an electric field along the axis of symmetry of the draping pattern, anti-sunward. Numerical models would be appropriate for investigating further this source of electric field, as was done for example by Huang et al. (2018) (though at a fairly different activity level). An additional source of pile-up for the magnetic field is the asymmetric Mach cone, the caustic of the model; therefore, $\boldsymbol{E}_{\mathrm{Hall}}$ is expected to play a role mostly in the downstream region of the caustic, tailward.

The third term, the ambipolar electric field $\boldsymbol{E}_{\text {ambipolar, }}$, is expected to be significant close to the nucleus. At zero order, this electric field points radially outward from the nucleus. It might be one of the reasons why close to the nucleus the modelled proton trajectories in Fig. 7 depart from the observed velocity vectors. Another effect arising from the ambipolar term is a polarisation electric field due to the different motion of the cometary new-born electrons and ions. The gyroradius of the cometary ions can be much larger than the interaction region, itself larger than the cometary electron gyroradius, which induces a charge separation, and in turn a polarisation electric field. This electric field contribution is explored in the analytical work of Nilsson et al. (2018), and results in one additional acceleration of the cometary ions with an anti-sunward component.

The three electric field are summarised in the schematic of Fig. 7. Above the nucleus $\left(z_{\mathrm{CSE}}>0\right), \boldsymbol{E}_{\text {motional }}$ and $\boldsymbol{E}_{\text {ambipolar }}$ are essentially aligned. Towards the inner tail region, $\boldsymbol{E}_{\text {ambipolar }}$ gives a tailward component to the total field compared to $\boldsymbol{E}_{\text {motional }}$ and eventually along the comet-Sun line, $\boldsymbol{E}_{\text {Hall }}$ adds up to the tailward acceleration of the cometary ions. Overall, the cometary ion acceleration ends up being mostly radial from the combination of the three terms in the generalised Ohm's law.

We note, however, that the apparent absence of acceleration for $r>600 \mathrm{~km}$ cannot be accounted for by this interpretation. Neither the ambipolar electric field nor the Hall term is expected to provide any significant acceleration there, but as this region is dominated by a barely perturbed solar wind the motional electric field is still about a few $\mathrm{mV} \mathrm{m}^{-1}$, as seen in the upper right panel in Fig. 7. An instrumental effect cannot be completely discarded, even though the energy and density ranges are nominal. The validation (or discussion) of this observation may require numerical modelling of the interaction, and we already note that the fully kinetic simulation presented by Deca et al. (2017) give a similar result, with the energy of the cometary ions increasing and reaching a plateau in the tail region (cf. Fig. 3 of Deca et al. 2017).

\subsection{Hybrid simulations}

A qualitatively identical dynamic is seen in Koenders et al. (2016), Fig. 14, lower and upper left panels, where the same interaction is simulated for a smaller heliocentric distance of $2.3 \mathrm{au}$. The solar wind forms an asymmetrical and curved Mach cone similarly to the caustic given by the semi-analytical model, and the overall deflection is in great qualitative agreement with both the data and the semi-analytical model. The simulated cometary ions are seen accelerated radially away from the nucleus on the night-side of the coma as well.

In another relevant simulation work, Bagdonat \& Motschmann (2002) describe a region of weak suppression of the magnetic field downstream of the nucleus and aligned with the comet-Sun line (Bagdonat \& Motschmann 2002, Fig. 4). The authors report a broad cycloidal tail leaving the nucleus orthogonal to the comet-Sun line (corresponding to our observation at $z>0$, above the nucleus), and a different cometary ion population accelerated tailward from the nucleus, corresponding to the observed cometary ion in the inner tail region.

\section{Summary and conclusions}

During the night-side excursion, the cometary ion flow and the solar wind remained very directional. The cometary beam appears quasi-radial, which is interpreted as the result of its acceleration by three different electric fields of different origins: the motion of the charge carriers, the electron pressure gradients, or the magnetic field line bending. The solar wind deflection is in agreement with the analytical expression of the the magnetic field pile-up and the motional electric field, with some possible influence of the two other electric field terms close to the nucleus and in the inner tail region.

A remaining open question is the apparent lack of acceleration of the cometary ions further than $700 \mathrm{~km}$ from the nucleus, which is not in agreement with the electric field components discussed previously.

The observed plasma dynamics on the night-side could enable better constraints in the comparisons between data and various models, as the area covered is much greater than usually available during the rest of the active mission. These better constraints would also allow us to direct more precisely the analysis of the simulation results as the range of parameters is significantly greater than has been explored in the present article. As an example, an obvious follow-up of this work would be the three-dimensional mapping of the different electric field terms in the result of a numerical simulation, which would enable the study of the interaction on the flanks of the coma away from the plane $\left(y_{\mathrm{CSE}}=0\right)$.

Acknowledgements. This work was supported by the Swedish National Space Board (SNSB) through grants 108/12, 112/13, and 96/15. The work at LPC2E/CNRS was supported by ESEP, CNES, and by ANR under the financial agreement ANR-15-CE31-0009-01. We acknowledge the staff of CDDP and IC for the use of AMDA and the RPC Quicklook database (provided by a collaboration between the Centre de Données de la Physique des Plasmas (CDPP) supported by CNRS, CNES, Observatoire de Paris and Université Paul Sabatier, Toulouse, and Imperial College, London, supported by the UK Science and Technology Facilities Council). We are indebted to the whole Rosetta mission team, Science Ground Segment, and Rosetta Mission Operation Control for their hard work making this mission possible.

\section{References}

Alfven, H. 1957, Tellus, IX, 92

Bagdonat, T., \& Motschmann, U. 2002, J. Comput. Phys., 183 
Behar, E. et al.: The root of a comet tail: Rosetta ion observations at comet $67 \mathrm{P} /$ Churyumov-Gerasimenko

Behar, E., Nilsson, H., Stenberg Wieser, G., et al. 2016, Geophys. Res. Lett., 43, 1411

Behar, E., Nilsson, H., Alho, M., Goetz, C., \& Tsurutani, B. 2017, MNRAS, 469, S396

Behar, E., Tabone, B., Saillenfest, M., et al. 2018, A\&A, DOI: $10.1051 / 0004-6361 / 201832736$

Berčič, L., Behar, E., Nilsson, H., et al. 2018, A\&A, 613, A57

Biermann, L. 1951, Z. Astrophys., 29, 274

Carr, C., Cupido, E., Lee, C. G. Y., et al. 2007, Space Sci. Rev., 128, 629

Coates, A., Burch, J., Goldstein, R., et al. 2015, J. Phys. Conf. Ser., 642, 012005

Deca, J., Divin, A., Henri, P., et al. 2017, Phys. Rev. Lett., 118, 205101

Durham, I. T. 2006, Notes and Records, 60, 261

Eddington, A. S. 1910, MNRAS, 70, 442

Glassmeier, K.-H., Boehnhardt, H., Koschny, D., Kührt, E., \& Richter, I. 2007, Space Sci. Rev., 128,

Haerendel, G., Paschmann, G., Baumjohann, W., \& Carlson, C. W. 1986, Nature, 320,720

Haser, L. 1957, Bull. Cl. Sci. Acad. Roy. Belg., 43, 740
Huang, Z., Tóth, G., Gombosi, T. I., et al. 2018, MNRAS, 475, 2835 Jones, G. H., Balogh, A., \& Horbury, T. S. 2000, Nature, 404, 574 Koenders, C., Perschke, C., Goetz, C., et al. 2016, A\&A, 594, A66 Nilsson, H., Lundin, R., Lundin, K., et al. 2007, Space Sci. Rev., 128, 671

Nilsson, H., Stenberg Wieser, G., Behar, E., et al. 2015, Science, 347

Nilsson, H., Wieser, G. S., Behar, E., et al. 2017, MNRAS, 469, S252

Nilsson, H., Gunell, H., Karlsson, T., etal. 2018, A\&A, in press, DOI: 10.1051/0004-6361/201833199

Richter, I., Koenders, C., Auster, H.-U., et al. 2015, Annales Geophysicae, 33, 1031

Saillenfest, M., Tabone, B., \& Behar, E. 2018, A\&A, in press, DOI: 10.1051/0004-6361/201832742

Slavin, J. A., Smith, E. J., Tsurutani, B. T., et al. 1986, Geophys. Res. Lett., 13, 283

Smith, E. J., Tsurutani, B. T., Slavin, J. A., et al. 1986, Science, 232, 382

Snodgrass, C., A'Hearn, M. F., Aceituno, F., et al. 2017, Phil. Trans. R. Soc. London, Ser. A, 375, 20160249

Valenzuela, A., Haerendel, G., Föppl, H., et al. 1986, Nature, 320, 700

Volwerk, M., Goetz, C., Richter, I., et al. 2018, A\&A, 614, A10 